\title{
Upregulation of proinflammatory genes in skin lesions may be the cause of keloid formation (Review)
}

\author{
XIANGLIN DONG ${ }^{1}$, SHAOLIN MAO ${ }^{1}$ and HAO WEN ${ }^{2}$ \\ ${ }^{1}$ Department of Burns and Plastic Surgery; ${ }^{2}$ Department of Hydatid Hepatobiliary, Digestive and Vascular Surgery Centre, \\ The First Affiliated Hospital of Xinjiang Medical University, Urumqi, Xinjiang 830011, P.R. China
}

Received August 29, 2013; Accepted September 19, 2013

DOI: $10.3892 /$ br.2013.169

\begin{abstract}
It was previously demonstrated that the main cause behind keloid formation may be keloid fibroblast abnormalities, which are closely associated with the microenvironment of the keloid lesion. The post-traumatic and chronic inflammation of the keloid lesion area suggest that inflammatory mediators play an important role in the keloid microenvironment and are crucial for keloid fibroblast abnormalities. In this study, we hypothesized that the mechanism underlying keloid formation may involve the continuous upregulation of proinflammatory gene expression in keloid lesions. This hypothesis may explain the inflammatory response, invasive growth and recurrence following resection of keloids, as well as the selective localization of keloids in specific parts of a patient's body and the differences in localization among different patients.
\end{abstract}

\section{Contents}

1. Introduction

2. Keloid fibroblast abnormalities may be the main cause of keloid formation

3. Role of the keloid lesion microenvironment in keloid formation

4. Chronic inflammation in keloids

5. Association of keloid formation with keloid fibroblasts, keloid lesion microenvironment and chronic inflammation

6. Proposed hypothesis for the cause of keloid formation

Correspondence to: Dr Shaolin Mao, Department of Burns and Plastic Surgery, The First Affiliated Hospital of Xinjiang Medical University, Urumqi, Xinjiang 830011, P.R. China

E-mail: mashaolin9@163.com

Dr Hao Wen, Department of Hydatid Hepatobiliary, Digestive and Vascular Surgery Centre, The First Affiliated Hospital of Xinjiang Medical University, Urumqi, Xinjiang 830011, P.R. China

E-mail: ycen99@yahoo.cn

Key words: proinflammatory genes, keloid formation, skin lesion

\section{Introduction}

Keloids are a type of benign, proliferative fibromas that form following trauma. The main characteristic of keloids is that they consist of fibroblasts exhibiting abnormally increased activity. Therefore, the connective tissue proliferates excessively with accompanying hyalinization, leading to overgrowth of scars. However, the pathogenesis has not been fully elucidated. Keloid scars are a type of unique benign human tumors without malignant potential that grow beyond their original boundaries and do not regress spontaneously, often resulting in organ deformity and dysfunction. Although independent or combined treatments may may be moderately effective, a meta-analysis revealed the treatment efficacy is not satisfactory $(1,2)$. Keloids commonly recur following resection. Furthermore, the transplantation of human keloids to mice indicated that keloids exhibit tumorigenic characteristics (3).

The mechanisms underlying keloid formation, invasive growth, recurrence following resection, selective localization and differences in localization among different patients have not been elucidated. The exact pathogenesis of keloids has not yet been determined (4), a fact that attracted the interest of numerous scholars and led to various hypotheses (5-8). However, thus far no hypothesis has provided sufficient explanation of the clinicopathological characteristics of keloids.

Previous clinical, histopathological and molecular studies revealed the presence of chronic inflammation in keloids, suggesting that trauma or local inflammation may lead to keloid formation (9-12). Therefore, we hypothesized that the proinflammatory genes in the keloid lesion area are stimulated by the inflammatory response in the microenvironment, leading to the formation of keloids. Issues such as the cause of keloid formation, invasive growth and recurrence following resection, as well as their clinical cahracteristics, may all be explained by our hypothesis.

\section{Keloid fibroblast abnormalities may be the main cause} of keloid formation

The biological basis of keloid formation lies in the substantial proliferation and apoptosis inhibition of fibroblasts, the imbalance between collagen synthesis and degradation in the extracellular matrix and the significantly elevated production of certain cytokines. It is known that the trauma repair 
process involves three stages: inflammation, granulation tissue formation and extracellular matrix formation (13). Following the early period of granulation tissue formation, the number of macrophages and fibroblasts is significantly increased. They continuously synthesize extracellular matrix and reshape the tissue through the contraction of granulation tissue. In the process of trauma repair, a balance is maintained for the control of fibroblast proliferation and extracellular matrix synthesis. An increase in cell proliferation with a concomitant reduction in apoptosis may lead to keloid formation (14). Most scholars believe that the abnormal proliferation of fibroblasts is the main cause of hyperplasia and persistence of pathological scars (15). Keloid fibroblasts are considered to proliferate more rapidly compared to the fibroblasts of hypertrophic scars (16), with oversecretion of type I collagen fibers and high expression levels of vascular endothelial growth factor, transforming growth factor- $\beta 1 / \beta 2$ and platelet-derived growth factor- $\alpha$ (17). Ladin et al (18) demonstrated that the apoptosis rate of keloid fibroblasts was relatively low, which was in accordance with the previously reported downregulation of apoptosis-related gene expression $(19,20)$. In addition, cultured keloid fibroblasts exhibited a distinct increase in collagen fiber synthesis and secretion of matrix metalloproteinase inhibitors compared to normal skin fibroblasts (21). Keloid fibroblasts also exhibited a significantly increased proliferative and anti-apoptotic ability compared to normal skin fibroblasts (22). It was suggested that the keloid fibroblast may be a pluripotent precursor cell with multiple differentiation capacities, similar to the human skin precursor cell, and may be able to differentiate into several types of cells, such as adipocytes, osteoblasts, chondrocytes, smooth muscle cells, vascular endothelial cells, glial cells and oligodendrocytes under different culture conditions (23). Studies using gene chip technology demonstrated an upregulation of $\sim 15 \%$ of the genes in keloid fibroblasts, among which the most important are proinflammatory factors, such as interleukin (IL)-1 $\alpha$, IL-1 $\beta$, IL-6 and tumor necrosis factor- $\alpha$ (TNF- $\alpha)(24,25)$. Based on those findings, keloid and normal skin fibroblasts are significantly different and keloid fibroblast abnormalities may be the main cause behind keloid formation.

\section{Role of the keloid lesion microenvironment in keloid formation}

Keloids usually recur following simple excision, due to the fact that the host fibroblasts surrounding the lesion develop into keloid fibroblasts within $24 \mathrm{~h}$ following keloid removal. Therefore, the majority of scholars advocate electronic radiotherapy within 24 or $48 \mathrm{~h}$ following keloid resection, which may be effective via the IL-6 pathway (26). Although keloid fibroblasts are similar to pluripotent human skin precursor cells, their use in cell replacement therapy (e.g., bones and nervous system), requires further investigation (27), as keloid fibroblasts lose certain characteristics in in vitro culture. While keloid fibroblasts share the same level of expression of $\alpha$ smooth muscle actin ( $\alpha$-SMA) with hypertrophic scar tissue in vitro, keloids rarely express $\alpha$-SMA in vivo (27). In addition, two different cell populations exhibiting $\alpha$-integrin expression were identified in keloids; however, the expression heterogeneity disappeared in a keloid fibroblast sub-culture (28).
Therefore, these characteristics of keloid fibroblasts are only present in the microenvironment of the keloid lesions. Thus, it may be concluded that the microenvironment of keloid lesions is vital for keloid formation. Accordingly, it is suggested that abnormal fibroblasts may be restored to normal if the cytokine microenvironment of keloids is manipulated $(27,28)$.

\section{Chronic inflammation in keloids}

Keloids mainly occur in positions such as anterior chest, lateral upper arm, scapular region and suprapubic area, with accompanying symptoms such as peripheral erythema, mild pain and pruritus, particularly during the early phases. The main histopathological characteristics include abnormal proliferation of fibroblasts, overaccumulation of extracellular matrix components such as glycoproteins, fiber adhesion factors and collagen and condensation of reticular collagen $(10,11)$. The center of the keloid lesion is highly collagenized, with an almost complete absence of blood vessels and cells. By contrast, a large number of lymphocytes and fibroblasts are dispersed among a rich network of blood vessels where the keloid is adjacent to normal skin $(27,29,30)$. Proliferation, apoptosis and necrosis coexist independently. The interplay between the invasive inflammatory cells in dermal tissue, vascular endothelial cells and fibroblasts may play a role in the excessive proliferation of keloids. During the process of keloid formation and development, angiogenesis is likely to play a significant role, since it supplies nutrients for keloid formation.

During the pathological process of wound healing or remodeling, fibroblasts are the main target and effector cells. Keloid fibroblasts secrete cytokines and chemokines, such as granulocyte-macrophage colony-stimulating factor, stem cell factor, TNF- $\alpha$, nerve growth factor, IL- 8 and eosinophil-activating factors. By using gene chip technology, Chen et al (24) and Messadi et al (19) discovered that $\sim 15 \%$ of genes in keloid fibroblasts, the most important being proinflammatory factors, were upregulated, suggesting that keloid fibroblasts are crucial in the occurrence of chronic inflammation. It was proposed that keloid formation may be caused by inflammation arising from the abnormal secretion of proinflammatory mediators and irregular response to other inflammatory signals mediated by keloid fibroblasts (12). It was also proven that, as a special form of wound healing, keloids exhibit characteristics similar to the long-lasting inflammatory responses, immune abnormalities and invasive growth characteristics of tumors $(31,32)$.

Nuclear transcription factor $-\kappa \mathrm{B}(\mathrm{NF}-\kappa \mathrm{B})$ is a protein family widespread in eukaryotes, which is composed of polypeptide subunits. Being involved in signal transduction pathways, it is essential for the transcription of a variety of proinflammatory genes. Upon activation, $\mathrm{NF}-\kappa \mathrm{B}$ may promote the mRNA expression and release of a variety of inflammatory mediators. It is considered that the $\mathrm{NF}-\kappa \mathrm{B}$ signaling pathway is activated in keloid tissues and fibroblasts, contributing to the persistence of keloid inflammation (19). In cultured keloid fibroblasts, $\mathrm{NF}-\kappa \mathrm{B}$ activity was increased compared to that in normal skin fibroblasts and the survival and development of keloid fibroblasts was shown to depend largely on NF- $\mathrm{BB}$ signal transduction (25). Accordingly, the inhibition of keloid formation by glucocorticoids may be mediated through inhibiting the activation of the NF- $\mathrm{B}$ signaling pathway (33). 
In addition, non-steroidal anti-inflammatory drugs, such as aspirin and sodium salicylate, may inhibit the binding of ATP to the inhibitor of NF- $\kappa \mathrm{B}$ kinase subunit $\beta$, thus inhibiting the $\mathrm{NF}-\kappa \mathrm{B}$ signaling pathway $(34,35)$.

Thus, the clinical, histopathological and molecular biological findings mentioned above indicate that chronic inflammation is present in keloids and plays an important role in the process of keloid formation.

\section{Association of keloid formation with keloid fibroblasts, keloid lesion microenvironment and chronic inflammation}

Keloid fibroblasts are mainly responsible for keloid formation, during which the microenvironment of the keloid lesion plays an important role in keloid fibroblast abnormalities. While trauma is the incentive for keloid formation, inflammatory mediators, such as cytokines and growth factors, being an important component of the keloid lesion microenvironment, play an important role in fibroblast abnormalities and keloid formation, since inflammatory responses are continuously present during the process of wound healing and keloid formation.

\section{Proposed hypothesis for the cause of keloid formation}

As discussed above, the keloid lesion microenvironment is mainly responsible for keloid fibroblast abnormalities and keloid formation. Various organisms have adjusted their own structure in order to adapt to the environmental changes during the long-term evolutionary process (survival of the fittest). Similarly, the human skin in different locations, such as the eyelids, dorsum of the body, face and foot, differs in its structure, in order to adapt to the different environmental requirements. Therefore, the local microenvironment of the skin and its susceptibility to external or internal stimuli differ by location. We hypothesized that the proinflammatory genes in the microenvironment of skin keloid lesions are highly sensitive to traumatic and post-traumatic inflammatory responses. The continuous upregulation of their expression following traumatic and inflammatory stimuli results in keloid formation and chronic inflammation.

It was previously proposed that keloid formation may result from inflammation arising from the secretion of proinflammatory mediators and the abnormal response to other inflammatory signals mediated by keloid fibroblasts (12). In accordance with this hypothesis, Chen et al (24) and Messadi et al (25) demonstrated that $\sim 15 \%$ of the genes, the most important being proinflammatory factors, such as IL-1 $\alpha$, IL-1 $\beta$, IL- 6 and TNF- $\alpha$, were upregulated in keloid fibroblasts.

Thus, the fact that proinflammatory genes in the microenvironment of the keloid skin lesions are sensitive to trauma and inflammatory response may explain the selective localization of keloids in certain parts of a patient's body and the differences in localization among different patients. This chronic inflammation may also explain the invasive growth of keloids.

The clinical treatment of keloids by glucocorticoid injection inhibits keloid formation mainly by repressing the activation of $\mathrm{NF}-\kappa \mathrm{B}$, an essential transcription factor of multiple proinflammatory genes (33). In addition, radiotherapy following keloid resection reduces keloid recurrence by blocking the IL-6 inflammatory response pathway (26). Thus, these treatments may help avoid the continuous upregulation of proinflammatory genes and reduce inflammation by blocking inflammatory response pathways, thus achieving satisfactory clinical results.

Based on the above, we concluded that the continuous upregulation of proinflammatory genes in the microenvironment of keloid skin lesions may be the main cause behind keloid formation.

\section{Acknowledgements}

This study was supported by the National Natural Science Foundation of China (no. 81260291). The authors would like to thank Dr Tao Liu and Dr Chuanshan Zhang at the laboratory of The First Affiliated Hospital of Xinjiang Medical University for their assistance.

\section{References}

1. Al-Attar A, Mess S, Thomassen JM, Kauffman CL and Davison SP: Keloid pathogenesis and treatment. Plast Reconstr Surg 117: 286-300, 2006.

2. Atiyeh BS, Costagliola M and Hayek SN: Keloid or hypertrophic scar: the controversy: review of the literature. Ann Plast Surg 54: 676-680, 2005.

3. Estrem SA, Domayer M, Bardach J and Cram AE: Implantation of human keloid into athymic mice. Laryngoscope 97: 1214-1218, 1987.

4. Pierce HE: Keloids: enigma of the plastic surgeon. J Natl Med Assoc 71: 1177-1180, 1979.

5. Huang C, Akaishi S, Hyakusoku $\mathrm{H}$ and Ogawa R: Are keloid and hypertrophic scar different forms of the same disorder? A fibroproliferative skin disorder hypothesis based on keloid findings. Int Wound J: Nov 22, 2012 (Epub ahead of print).

6. de Mesquita CJ: About strawberry, crab claws, and the Sir James Black's invention. Hypothesis: can we battle keloids with propranolol? Med Hypotheses 74: 353-359, 2010.

7. Akaishi S, Ogawa R and Hyakusoku H: Keloid and hypertrophic scar: neurogenic inflammation hypotheses. Med Hypotheses 71: 32-38, 2008.

8. Alonso PE, Rioja LF and Pera C: Keloids: A viral hypothesis. Med Hypotheses 70: 156-166, 2008.

9. O'Brien L and Jones DJ: Silicone gel sheeting for preventing and treating hypertrophic and keloid scars. Cochrane Database Syst Rev 9: Sep 12, 2013 (Epub ahead of print).

10. Kischer CW, Shetlar MR and Chvapil M: Hypertrophic scars and keloids: a review and new concept concerning their origin. Scan Electron Microsc 4: 1699-1713, 1982.

11. Tredget EE, Nedelec B, Scott PG and Ghahary A: Hypertrophic scars, keloids, and contractures. The cellular and molecular basis for therapy. Surg Clin North Am 77: 701-730, 1997.

12. Sandulache VC, Parekh A, Li-Korotky H, Dohar JE and Hebda PA: Prostaglandin E2 inhibition of keloid fibroblast migration, contraction, and transforming growth factor (TGF)-beta 1-induced collagen synthesis. Wound Repair Regen 15: 122-133, 2007.

13. Clark RA: Cutaneous tissue repair: basic biologic considerations. I. J Am Acad Dermatol 13: 701-725, 1985.

14. Sahl WJ Jr and Clever H: Cutaneous scars: Part I. Int J Dermatol 33: 681-691, 1994.

15. Nakaoka H, Miyauchi S and Miki Y: Proliferating activity of dermal fibroblasts in keloids and hypertrophic scars. Acta Derm Venereol 75: 102-104, 1995

16. Nedelec B, Shankowsky H, Scott PG, Ghahary A and Tredget EE: Myofibroblasts and apoptosis in human hypertrophic scars: the effect of interferon-alpha2b. Surgery 130: 798-808, 2001.

17. Marneros AG and Krieg T: Keloids - clinical diagnosis, pathogenesis, and treatment options. J Dtsch Dermatol Ges 2: 905-913, 2004.

18. Ladin DA, Hou Z, Patel D, et al: p53 and apoptosis alterations in keloids and keloid fibroblasts. Wound Repair Regen 6: 28-37, 1998. 
19. Messadi DV, Le A, Berg S, et al: Effect of TGF-beta 1 on PDGF receptors expression in human scar fibroblasts. Front Biosci 3: a16-a22, 1998.

20. Sayah DN, Soo C, Shaw WW, et al: Downregulation of apoptosis-related genes in keloid tissues. J Surg Res 87: 209-216, 1999.

21. Fujiwara M, Muragaki Y and Ooshima A: Keloid-derived fibroblasts show increased secretion of factors involved in collagen turnover and depend on matrix metalloproteinase for migration. Br J Dermatol 153: 295-300, 2005.

22. Calderon M, Lawrence WT and Banes AJ: Increased proliferation in keloid fibroblasts wounded in vitro. J Surg Res 61: 343-347, 1996.

23. Moon JH, Kwak SS, Park G, et al: Isolation and characterization of multipotent human keloid-derived mesenchymal-like stem cells. Stem Cells Dev 17: 713-724, 2008.

24. Chen W, Fu X, Sun X, et al: Analysis of differentially expressed genes in keloids and normal skin with cDNA microarray. J Surg Res 113: 208-216, 2003

25. Messadi DV, Doung HS, Zhang Q, et al: Activation of NFkappaB signal pathways in keloid fibroblasts. Arch Dermatol Res 296 125-133, 2004.

26. Tosa M, Ghazizadeh M, Shimizu H, et al: Global gene expression analysis of keloid fibroblasts in response to electron beam irradiation reveals the involvement of interleukin-6 pathway. J Invest Dermatol 124: 704-713, 2005.

27. Ehrlich HP, Desmouliere A, Diegelmann RF, et al: Morphological and immunochemical differences between keloid and hypertrophic scar. Am J Pathol 145: 105-113, 1994.
28. Szulgit G, Rudolph R, Wandel A, et al: Alterations in fibroblast alphalbeta1 integrin collagen receptor expression in keloids and hypertrophic scars. J Invest Dermatol 118: 409-415, 2002.

29. Alster TS and Tanzi EL: Hypertrophic scars and keloids: etiology and management. Am J Clin Dermatol 4: 235-243, 2003.

30. Tuan TL and Nichter LS: The molecular basis of keloid and hypertrophic scar formation. Mol Med Today 4: 19-24, 1998.

31. Zhang Q, Oh CK, Messadi DV, et al: Hypoxia-induced HIF-1 alpha accumulation is augmented in a co-culture of keloid fibroblasts and human mast cells: involvement of ERK1/2 and PI-3K/Akt. Exp Cell Res 312: 145-155, 2006.

32. Harty M, Neff AW, King MW and Mescher AL: Regeneration or scarring: an immunologic perspective. Dev Dyn 226: 268-279, 2003.

33. Makino S, Mitsutake N, Nakashima M, et al: DHMEQ, a novel NF-kappaB inhibitor, suppresses growth and type I collagen accumulation in keloid fibroblasts. J Dermatol Sci 51: 171-180, 2008.

34. De Bosscher K, Schmitz ML, Vanden Berghe W, et al: Glucocorticoid-mediated repression of nuclear factor-kappaBdependent transcription involves direct interference with transactivation. Proc Natl Acad Sci USA 94: 13504-13509, 1997.

35. Yin MJ, Yamamoto $Y$ and Gaynor RB: The anti-inflammatory agents aspirin and salicylate inhibit the activity of IкB kinase- $\beta$. Nature 396: 77-80, 1998.

36. Gilmore TD and Herscovitch M: Inhibitors of NF-kappaB signaling: 785 and counting. Oncogene 25: 6887-6899, 2006. 